\title{
Politique
}

Politique

\section{A. Cambrosio et R. Duchesne (sous la direction de), Science, technologie et Société. Les enjeux du progrès, Sainte-Foy, Presses de l’Université du Québec et Télé-université, 1984, $356 \mathrm{p}$.}

\section{Réjean Landry}

Numéro 8, automne 1985

Innovations et politiques technologiques

URI : https://id.erudit.org/iderudit/040501ar

DOI : https://doi.org/10.7202/040501ar

Aller au sommaire du numéro

\section{Éditeur(s)}

Société québécoise de science politique

ISSN

0711-608X (imprimé)

1918-6584 (numérique)

Découvrir la revue

Citer ce compte rendu

Landry, R. (1985). Compte rendu de [A. Cambrosio et R. Duchesne (sous la direction de), Science, technologie et Société. Les enjeux du progrès, Sainte-Foy, Presses de l'Université du Québec et Télé-université, 1984, 356 p.] Politique, (8), 144-146. https://doi.org/10.7202/040501ar d'utilisation que vous pouvez consulter en ligne. 
A. Cambrosio et R. Duchesne (sous la direction de), Science, technologie et Société. Les enjeux du progrès, Sainte-Foy, Presses de l'Université du Québec et Télé-université, 1984, 356 pages.

Il s'agit d'un recueil de douze textes conçu pour les étudiants de premier cycle de la Télé-université (Université du Québec). Le recueil est constitué de textes bien documentés dont plusieurs, particulièrement ceux qui ont été publiés ailleurs, commencent à dater considérablement. De plus, contrairement à ce que suggère le graphique illustrant la page titre, la plupart des textes abordent la question de la science, de la technologie et de la société suivant une perspective historique plutôt que de traiter de leurs aspects économiques. Il ne s'agit pas d'un ouvrage d'analyse de politiques mais d'une réflexion «humaniste» sur le vaste thème que recouvrent les notions de «science, technologie et société».

Le texte le mieux documenté du recueil, celui de C. Freeman sur l'économique de la recherche et du développement (chapitre 1) a été publié pour la première fois en 1977. Son apport le plus intéressant concerne l'histoire de la pensée des économistes sur le progrès technique car il est devenu grandement dépassé du côté des résultats de la recherche empirique aussi bien que de celui des repères bibliographiques. Le chapitre 2 intitulé "Développements techniques, travail humain structure sociale» constitue un texte original de R. Duchesne. Ce chapitre analyse la relation machine/travail en étudiant l'impact de la mécanisation et de l'automation sur la nature du travail humain. Cette question est traitée d'un point de vue historique en adoptant le mode de l'illustration plutôt que celui de la démonstration rigoureuse. Le texte de F. Sandback sur la genèse et le déclin du débat sur les limites de la croissance à été publié pour la première fois en 1978. Il est difficile de comprendre les raisons qui justifient l'inclusion de ce texte dans le recueil puisqu'il relate l'histoire d'une problématique des années soixante-dix qui suscite actuellement beau- 
coup moins d'intérêt. Les chapitres 4 sur la politique scientifique canadienne et 5 sur la politique scientifique du Québec constituent des contributions originales de R. Duchesne. Il s'agit peut-être des deux chapitres les plus faibles de tout le recueil: l'auteur adopte une perspective historique et, lorsqu'il aborde les débats actuels, il le fait généralement en référant à des documents et à des données empiriques qui ne sont pas à jour. La thèse du texte de E. Colombino sur «science, technologie et Tiers-Monde» (chapitre 6), qui est fort complexe, peut être résumée en deux points: la science et la technique ont engendré l'émergence du capitalisme et stimulé son expansion mondiale; cette expansion mondiale du capitalisme a engendré une crise structurelle dans les pays du Tiers-Monde. Le texte de R. C. Lewontin sur «le déterminisme biologique comme arme sociale» (chapitre 7) ne concerne pas l'univers des sujets que recouvre le vaste thème «science, technologie et société». Le chapitre 8 de D. Nelkin analyse les conséquences de la participation des scientifiques aux débats dans des domaines controversés. Il s'agit d'un texte fort intéressant publié pour la première fois en 1975 par une chercheure qui a publié plus récemment beaucoup d'autres textes sur le même sujet. Le texte de D. J. Roy (chapitre 9) sur la bioéthique se veut plus informatif qu'analytique: il comporte plus d'informations sur les sujets qui intéressent la bioéthique que sur sa façon (théories et méthodes) de traiter les sujets. C. Halary (chapitre 10) trace un bref mais intéressant historique des concepts d'automates et de robots. Le chapitre 11, rédigé par $\mathrm{M}$. Fournier fait l'historique, non pas comme le dit le titre, du procès des sciences sociales mais de la sociologie. Certaines remarques de Fournier débordent toutefois le champ de la sociologie: d'après celui-ci les sciences sociales constituent des technologies sociales, «c'est-à-dire des moyens de gérer d'une manière instrumentale des ressources sociales et humaines, et de contrôler le fonctionnement de la société. Étroitement associées à cette modernisation et à cette rationalisation de la 
gestion publique, les sciences sociales peuvent difficilement échapper à la critique à partir du moment où l'État lui-même et son mode de fonctionnement sont l'objet d'une remise en question.» (p. 331). Finalement le chapitre 12 sur la vulgarisation scientifique a été publié pour la première fois par $\mathrm{B}$. Jurdant dans la revue $\mathrm{La}$ recherche en 1975.

Dans l'ensemble, le contenu du recueil ne reflète pas ce qu'annonce le titre. L'approche historique est privilégiée au détriment de l'analyse critique des politiques actuelles. Le recueil comporte des trous béants: ainsi, on n'y retrouve aucun texte sur l'évaluation technologique et sur l'évaluation des interventions gouvernementales. En résumé, ce recueil de textes ne rend pas compte de l'état actuel des connaissances sur le thème «science, technologie et société».

Réjean Landry

Université Laval 